\title{
Influência da Idade, Desejabilidade Social e Memória na Sugestionabilidade Infantil
}

\author{
Influence of Age, Social Desirability and Memory in Children's Suggestibility
}

\author{
Magda Saraiva \& Pedro B. Albuquerque* \\ Escola de Psicologia, Universidade do Minho, Braga, Portugal
}

\begin{abstract}
Resumo
A sugestionabilidade infantil é influenciada pela capacidade de memória, idade ou desejabilidade social. Este estudo teve como objetivo estudar estes fatores em três grupos de crianças com idades de 5/6 anos, 8/9 anos e 10/11 anos. Para tanto procedemos à construção de uma escala de avaliação da sugestionabilidade infantil baseada na Escala de Sugestionabilidade de Gudjonsson (GSS; Gudjonsson, 1997). Os resultados obtidos revelaram que as crianças mais novas foram as mais sugestionáveis, fenômeno que parece estar associado ao elevado grau de desejabilidade social das suas respostas. Relativamente à memória fonológica, verificamos que a sua menor amplitude esteve também relacionada com maior sugestionabilidade infantil. Sugere-se que as variáveis memória e desejabilidade social sejam cuidadosamente consideradas no momento de obtenção do testemunho de crianças.

Palavras-chave: Sugestionabilidade infantil, memória, desejabilidade social, testemunho infantil.
\end{abstract}

\begin{abstract}
Children's suggestibility is influenced by several factors such as memory, age or social desirability. The main objective of this study was to analyze how these factors are associated with the degree of suggestibility in 5/6 year-old, 8/9 year-old and 10/11 year-old children. For a suggestibility measure, a new scale based on the Gudjonsson Suggestibility Scale was developed (GSS; Gudjonsson, 1997). Results showed that: younger children are more suggestible than older children; a higher degree of social desirability is associated with stronger suggestibility responses; and a smaller memory span is related to greater children's suggestibility. Conclusions highlight that memory and social desirability should be analyzed carefully when eyewitness accounts of children are considered.
\end{abstract}

Keywords: Children's suggestibility, memory, social desirability, child witness.

A credibilidade dos relatos baseados nas nossas memórias e a veracidade fatual das memórias da infância tem levado a Psicologia Forense a interessar-se cada vez mais pelo estudo da credibilidade dos testemunhos em geral, e das crianças em particular. Nesse cenário, e também devido ao interesse crescente pelo estudo das memórias falsas, muito se tem especulado e investigado acerca da sugestionabilidade em crianças.

Compreender o funcionamento da memória passa por perceber os efeitos que o decurso do tempo tem sobre o esquecimento de acontecimentos vividos, mas também sobre a sua distorção. Dessa forma, quanto mais tempo decorre entre um acontecimento e a sua recordação, mais informação se esquece ou distorce, tornando-se, com passar do tempo, as memórias do acontecimento cada vez menos detalhadas ou pormenorizadas (Roediger \& McDermott, 1995; Schacter, 1999). Relativamente às distorções de memória em crianças esse dado é ainda mais saliente e eloquente (Costa \& Pinho, 2010).
A Psicologia Cognitiva Forense tem desenvolvido esforços de modo a compreender e construir instrumentos e entrevistas adequadas às crianças, medidas que possibilitem a obtenção de relatos menos sujeitos à sugestão e, por força disso, mais credíveis. Este é um dos objetivos desse nosso artigo.

\section{O Conceito de Sugestionabilidade}

Por força do recente estudo da sugestionabilidade infantil não existe consenso quanto à definição de um conceito único. Ainda assim, os autores concordam que a sugestionabilidade pode ser entendida como uma disposição psicológica das pessoas para seguirem uma indicação fornecida por alguém ou sugerida por um acontecimento, integrando essa sugestão na sua história pessoal ou agindo em conformidade com ela (Gudjonsson, 1997). Também Schacter (2003) vai ao encontro dessa definição ao sugerir que a sugestionabilidade é a tendência individual para integrar informações distorcidas nas nossas memórias pessoais. Numa outra perspectiva, Gudjonsson e Clark (1986), definem esse fenômeno como estando restrito ao modo como alteramos nosso comportamento a partir da aceitação de mensagens veiculadas numa interação social. 
Atualmente a sugestionabilidade é entendida como o modo como fatores sociais, individuais e psicológicos influenciam a retenção, codificação e recuperação de determinados acontecimentos (Bruck \& Melnyk, 2011; Ceci $\&$ Bruck, 1993). Em outras palavras, a sugestionabilidade é vista como o resultado de múltiplos fatores, e esta multidimensionalidade é ainda mais marcante quando lidamos com a sugestionabilidade em crianças (para uma revisão ver Bruck \& Melnyk, 2011).

Mas afinal que mecanismos suportam este fenômeno? Serão as crianças sugestionáveis por força da desejabilidade social presente no momento de responder ao entrevistador? Ou será para se moldarem a uma pessoa que é percebida como fonte de autoridade? Será que as crianças ao repetirem várias vezes o mesmo relato distorcido de uma vivência alteram os traços de memória associados a essa vivência?

Para Ceci e Bruck (1993), as crianças são sugestionáveis devido à sua vulnerabilidade à pressão social, isso é, para esses autores, as crianças confiam nos adultos e por isso tendem a dar respostas que julgam agradar-lhes, independentemente da sua veracidade ou precisão. Esse fundamento justifica o fato de as crianças alterarem sua resposta, ao serem questionadas duas vezes acerca do mesmo episódio, uma vez que interpretam a repetição da questão como uma segunda oportunidade, ou um sinal para responderem de modo mais correto. Ainda assim, essa alteração da resposta pode não se consolidar como um traço de memória. Com efeito, Cassel, Roebers e Bjorklund (1996), mostraram que as crianças são mais sugestionáveis do que os adultos, no entanto quando questionadas alguns anos mais tarde sobre o mesmo fato, são capazes de relatar a história original de modo preciso e não sugestivo.

\section{O Estudo da Sugestionabilidade Infantil}

No centro da investigação da sugestionabilidade infantil está o paradigma da desinformação. Este paradigma é ainda hoje amplamente utilizado no estudo da sugestionabilidade infantil, pois permite examinar diretamente o efeito da introdução de questões sugestivas nas respostas das crianças.

Com origem em 1974, e proposto por Loftus e Palmer, o paradigma da desinformação foi inicialmente criado com recurso à apresentação de um filme sobre um acidente automobilístico. Imediatamente após a apresentação dessas imagens, os sujeitos são instruídos no sentido de descreverem, com pormenor, tudo aquilo de que se recordam. Depois são interrogados acerca do acontecimento por meio de um questionário que inclui questões sugestivas, tais como: "A que velocidade circulavam os automóveis quando chocaram um no outro?". Essa questão tem um cariz sugestivo presente na manipulação intencional da intensidade do verbo "chocar" (por oposição a "esmagar") podendo sugerir ou ajudar a referenciar a estimativa de velocidade (Loftus \& Palmer, 1974). As restantes questões sugestivas, manipuladas de forma interparticipante, variavam apenas nos verbos utilizados, tendo os autores apresentado as expressões "colidir" e "esmagar".

Loftus e Palmer (1974) demonstraram que a introdução de questões sugestivas leva a que as estimativas de velocidade sejam influenciadas pela intensidade do verbo, mas também a que os participantes desenvolvam relatos menos precisos sobre os materiais apresentados, já que uma semana mais tarde os participantes descrevem detalhes e pormenores que nunca visualizaram nos diapositivos (e.g., vidros quebrados no chão). Esse estudo é um dos marcos na demonstração de como uma simples questão pode alterar a recuperação mnésica.

Na década de 1980 os investigadores optaram, na sua maioria, por um método de estudo que consiste em expor as crianças a questões sugestivas ou que contêm premissas falsas acerca de uma história visualizada ou contada. Utilizando esse método, Ceci e Bruck (1993) concluíram que as crianças mais novas são muito sugestionáveis, fenômeno esse que tende a diminuir com o avançar da idade. Recentemente, Goswick, Mullet e Marsh (2013) sugeriram que as crianças mais velhas são mais sugestionáveis do que as mais novas, devido à capacidade e tendência destas para interpretarem informações da história apresentada, com base no seu conhecimento geral prévio, o que as conduz ao erro.

Outra variável a ter em conta na sugestionabilidade é a influência do entrevistador. Segundo Ceci e Bruck (1995) e Ceci e Friedman (2000), as convicções do entrevistador influenciam o modo como esses questionam as crianças, podendo usar um tom acusatório ou defensivo, o que aumenta a sugestionabilidade. O estudo de Almerigogna, Ost, Akehurst e Fluck (2008), teve como objetivo compreender como o comportamento não verbal dos entrevistadores afeta a sugestionabilidade em crianças com 8 e 10 anos. Os resultados revelaram que as crianças entrevistadas por entrevistadores considerados menos empáticos (e.g., comportamento de inquietação durante a entrevista), foram menos precisas na resposta às questões. Outros autores referem que a influência das expectativas pode ser gerada pelas próprias crianças. Um estudo recente descreveu um efeito de auto-sugestionabilidade que resulta de processos internos que conduzem a distorções de memórias sugeridas pelas expectativas, crenças e objetivos das próprias crianças (Principe \& Schindewolf, 2012).

Em Portugal o estudo da sugestionabilidade infantil está ainda no seu início. A esse nível, destaca-se o estudo de Cunha (2010), que adaptou duas escalas de avaliação da sugestionabilidade: a Escala de Sugestionabilidade de Gudjonsson (GSS2; Gudjonsson, 1987) e a Escala de Sugestionabilidade em Vídeo para Crianças (VSSC; Scullin \& Ceci, 2001). Ao comparar desempenhos de crianças de 7 e 12 anos nas escalas GSS e VSSC a autora concluiu que as crianças mais novas são mais sugestionáveis do que as crianças mais velhas.

Estudos em que são comparadas faixas etárias na resposta a instrumentos de sugestionabilidade são os mais representados na literatura. Contudo, variáveis como a 
capacidade de memória (Cunha, 2010; Henry \& Gudjonsson, 2003), o domínio da linguagem (Scullin, Kanaya, \& Ceci, 2002), a relação com as funções executivas (Karpinski \& Scullin, 2009), a desejabilidade social na resposta (Costa \& Pinho, 2010) ou a pressão exercida por uma figura de autoridade/adulto (Okanda, Kanda, Ishiguro, \& Itakura, 2013) têm também sido estudados.

Também no Brasil, o estudo da sugestionabilidade infantil está no começo. Devido a crescente presença de crianças em tribunal como testemunhas, foram desenvolvidas algumas reflexões para perceber a capacidade das crianças para recordar experiências, acontecimentos ou situações e o modo como essas memórias são ou não confiáveis (e.g., Stein, Pergher, \& Feix, 2009; Welter \& Feix, 2010). Para tanto, os referidos autores têm orientado seus estudos no sentido de compreender quais os fatores individuais, desenvolvimentais e externos que afetam a sugestionabilidade. Assim, os seus resultados têm demonstrado que as crianças mais novas são de fato mais sugestionáveis. Os autores defendem ainda que é entre os 2 e 5 anos de idade que a sugestionabilidade é mais evidente, e apontam a fraca capacidade mnésica das crianças dessas idades como um dos fatores que mais contribui para esse resultado. Por outro lado, à semelhança de outros autores (e.g., Cunha, 2010), referem que a inteligência verbal e as habilidades linguísticas são inversamente proporcionais à sugestionabilidade infantil. Finalmente, o contexto de entrevista (e.g., sala de audiências do tribunal), as expectativas do investigador face às respostas da criança, o tom emocional da entrevista, bem como o número de entrevistas aumentam o grau de sugestionabilidade infantil (Welter \& Feix, 2010).

No que diz respeito ao estudo da influência da memória episódica na sugestionabilidade infantil, o foco se centra na seguinte questão: será a memória para acontecimentos importantes um preditor de maior resistência à sugestionabilidade? Segundo Liebman e colaboradores (2002), existe uma correlação negativa entre a capacidade de memória episódica e sugestionabilidade, uma vez que, quanto melhor uma criança recorda um determinado acontecimento, mais resistente é à sugestão, associação que também é defendida por Candel, Merckelbach, Jelicic, Limpens e Widdershoven (2004). No entanto outros autores referem que a capacidade de memória das crianças não é um bom preditor do grau de sugestionabilidade que apresentam (Cunha, 2010). A memória parece ter um papel importante apenas quando falamos sobre a capacidade de recordação do material ou informação sobre a qual a sugestão vai incidir.

Por outro lado, a desejabilidade social tem também sido estudada na sua relação com a sugestionabilidade infantil (e.g., Costa \& Pinho, 2010). Para esses estudos importa perceber se a tendência para responder de forma socialmente desejável é uma característica pessoal associada à também maior tendência para, por exemplo, responder afirmativamente às questões sugestivas. Dessa forma, várias escalas que avaliam a desejabilidade social têm sido construídas e aplicadas, como é o exemplo do Questionário de Desejabilidade Social para Crianças (CSD; Crandall, Crandall, \& Katkovsky, 1965). A aplicação do CSD tem revelado que a desejabilidade social é inversamente proporcional à idade cronológica das crianças, pois as crianças mais novas fornecem mais respostas socialmente desejáveis do que as crianças mais velhas, variação que parece ser paralela ao padrão de resultados encontrados na sugestionabilidade.

Assim, esse artigo tem como objetivos principais: demonstrar o desempenho na Escala de Sugestionabilidade Interrogativa (ESI) por diferentes grupos etários, levando em consideração a memória fonológica, e, estabelecer relações entre a ESI e desejabilidade social.

\section{Método}

\section{Participantes}

Participaram nesse estudo 70 crianças, sendo 35 do sexo masculino e 35 do sexo feminino. A idade das crianças variou entre os 62 e os 142 meses $(M=102,9, D P=$ $22,49)$ e a sua distribuição pelas três faixas etárias foi a seguinte: $16(22,9 \%)$ tinham 5/6 anos; 35 (50\%) tinham $8 / 9$ anos; e $19(27,1 \%)$ tinham 10/11 anos. Todos os dados foram recolhidos numa escola privada do Concelho de Braga, Portugal.

A participação no estudo foi voluntária, sendo os pais/ responsáveis pelas crianças informados do seu objetivo e procedimentos, tendo para isso assinado um consentimento informado.

\section{Desenho Experimental}

O nosso estudo procurou relacionar a idade das crianças com o nível de sugestionabilidade interrogativa medido através da ESI (Saraiva \& Albuquerque, 2012), que é uma adaptação da Escala de Sugestionabilidade de Gudjonson (Gudjonsson, 1997).

\section{Instrumentos}

Escala de Sugestionabilidade Interrogativa (adaptação de Saraiva \& Albuquerque, 2012). Para a concretização deste estudo decidimos realizar uma adaptação da Escala de Sugestionabilidade de Gudjonsson (GSS; Gudjonsson, 1997). Essa adaptação teve como foco principal a criação de um instrumento cuja história fosse mais próxima da realidade vivida por crianças; e na formulação de um questionário que se orientou pelos mesmos quatro tipos de questões propostas por Gudjonsson (1997).

$\mathrm{Na}$ adaptação da história se optou pela ausência de conteúdo criminal e por uma dimensão textual próxima da GSS (a nossa história apresenta 44 unidades de informação, cf. Anexo). Seguindo o modelo de Gudjonsson, foi também elaborado um questionário com 20 questões ${ }^{1}$, sendo 
5 verdadeiras (1, 5, 9, 13, 17 - e.g., "O Simão e o André são irmãos?”), 5 com duas alternativas falsas (2, 6, 10, 14,18 - e.g., "A casa dos irmãos é branca ou amarela?”), 5 afirmativas (3, 7, 11,15, 19 - e.g., "A mãe dos rapazes é professora?") e 5 capciosas (4, 8, 12, 16, 20 - e.g., "Os dois rapazes são saudáveis?").

$\mathrm{O}$ procedimento de administração da ESI é idêntico ao proposto na GSS de Gudjonsson (1997) e será descrito adiante.

Tal como ocorre na GSS, essas escalas permitem 4 tipos de medida: "submissão 1", "submissão 2", "mudança" e "sugestionabilidade total". Dessa forma, a pontuação na medida de submissão 1 corresponde ao número de respostas afirmativas às 15 questões sugestivas na primeira administração do questionário; por outro lado, a medida de submissão 2 corresponde ao mesmo tipo de resposta na segunda administração do mesmo questionário. Essas medidas têm uma pontuação máxima de 15 pontos.

A variável dependente mudança diz respeito ao número de respostas da segunda administração do questionário, respondido após a introdução do feedback negativo, cujas respostas foram diferentes das emitidas na primeira administração. Essa medida tem a pontuação máxima de 20 pontos, pois todas as questões (sugestivas ou não) podem estar sujeitas a mudança de resposta.

Por fim, a medida de sugestionabilidade total é conseguida através do somatório das medidas de submissão1 e mudança, permitindo um resultado máximo de 35 pontos.

Questionário de Desejabilidade Social para Crianças (Children's Social Desirability - Crandall et al., 1965, versão traduzida por Costa \& Veloso 2008). Essa escala avalia o grau em que a criança responde a questões ou ações de forma conveniente com o que se espera dela ou do que ela julga ser mais aceitável pelos outros. Essa versão reduzida da escala apresenta apenas 14 itens e foi objeto de tradução e adaptação para a população portuguesa por Costa e Veloso (2008).

$\mathrm{Na}$ versão reduzida da CSD apenas são permitidas respostas do tipo sim/não aos 14 itens que a constituem. Para quatro destes 14 itens espera-se que a desejabilidade social da resposta leve as crianças a emitirem uma resposta afirmativa (e.g., Você se preocupa sempre em ter as suas roupas limpas e o seu quarto arrumado?) e aos restantes 10 itens se espera uma resposta negativa (e.g., Já alguma vez sentiu que disse coisas menos simpáticas a alguém?). Para avaliar a desejabilidade social, que varia entre 0 e 14 (número de itens), são somadas as respostas dadas pelas crianças que vão ao encontro das respostas esperadas ou socialmente desejáveis.

Prova de Evocação de Pseudopalavras (PEPp; versão de Vicente, 2003). Trata-se de uma prova que avalia a

dois factos ou pormenores contraditórios com a história apresentada; as questões afirmativas não têm premissas falsas salientes mas que podem ser capciosas porque geram a dúvida no respondente; e, finalmente as questões capciosas incluem na sua formulação pelo menos uma premissa que gera a expectativa de uma determinada resposta. amplitude de memória fonológica das crianças em tarefas de memória de curto prazo.

Essa prova é baseada no teste de pseudopalavras proposto na bateria de memória operacional Working Memory Test Battery for Children (WMTB-C; Pickering \& Gathercole, 2001). A PEPp constituída por uma lista de pseudopalavras, isso é, palavras que não fazem parte do léxico do Português Europeu, mas que apresentam uma estrutura lexical semelhante (e.g., Raca, Dola, etc.). Essa prova está dividida em seis blocos de pseudopalavras e cada bloco corresponde a uma extensão ou unidade de amplitude de memória fonológica. A primeira extensão tem a amplitude de uma unidade e é constituída por seis sequências que são apresentadas uma de cada vez. Para essa extensão, após a apresentação de uma pseudopalavra, a criança deve recordá-la corretamente. A segunda extensão tem uma amplitude de duas unidades e, nesse caso, são apresentadas duas pseudopalavras ao ritmo de 1 segundo por pseudopalavra e a criança deve recordá-las na ordem em que foram apresentadas. As restantes extensões seguem o mesmo padrão procedimental.

As palavras são apresentadas pelo investigador num tom de voz constante e claro e ao ritmo de uma pseudopalavra por segundo. Na administração da prova tem-se em conta que, para cada extensão, assim que a criança evoca corretamente quatro dos seis ensaios de pseudopalavras previstos para cada extensão, avança-se para a extensão seguinte. Quando a criança não é capaz de recordar corretamente quatro dos seis ensaios de uma extensão, a prova é interrompida, correspondendo a amplitude de memória da criança à última extensão em que foi bem sucedida.

No que diz respeito à pontuação dessa prova, um acerto é pontuado com 1 e um erro com 0 pontos. Dessa forma, a pontuação máxima é 36 (6 sequências de pseudopalavras X 6 amplitudes ou extensões possíveis).

Aplicações de provas de evocação de pseudopalavras nos mostram que as crianças mais novas possuem uma amplitude de memória mais baixa do que crianças mais velhas (Lobo, Acrani, \& Ávila, 2008). Dessa forma, se espera que crianças com maior amplitude de memória fonológica sejam mais resistentes à sugestionabilidade.

\section{Procedimento}

Os instrumentos usados nesse estudo foram administrados individualmente numa sala. Para a concretização desse estudo, começamos por aplicar a ESI para avaliar a sugestionabilidade infantil. Nesse sentido, as crianças ouviram a história (lida pela experimentadora), sendo instruídas a prestarem atenção, pois deveriam contá-la mais tarde. No final da história, foi pedido às crianças para a relatarem em voz alta (evocação 1). Seguiu-se um intervalo de aproximadamente 30 minutos, ocupados com a administração do Questionário de Desejabilidade Social para Crianças e a Prova de Evocação de Pseudopalavras segundo o procedimento anteriormente descrito.

No final dessas provas, e tendo assegurado que a sua resposta demora 30 minutos, as crianças foram de novo 
instruídas a contar com detalhe a história inicialmente apresentada (evocação 2). Deve-se salientar que tanto a evocação 1 como a evocação 2 foram gravadas em áudio, de modo a permitir sua transcrição e posterior contabilização das unidades de informação evocadas por cada criança em cada um dos dois momentos. Para efeito da contabilização das unidades de informação, apenas foram tidas em conta as recordações literais, não sendo contabilizados os sinônimos.

Quando as crianças não recordavam mais nenhuma informação da história, foi administrado, pela primeira vez, o questionário sobre os conteúdos da ESI (cf. Anexo). Em seguida foi dito às crianças que erraram muitas respostas ao questionário (introdução do feedback negativo) e que por isso se iria proceder a uma segunda administração, o que foi feito imediatamente.

O procedimento termina com uma explicação a cada criança dos principais objetivos do estudo. $O$ procedimento teve uma duração média de 45 minutos.

\section{Resultados}

\section{Análise Estatística}

A variável idade foi manipulada de forma inter-participante e apresentou três níveis: 5/6 anos; 8/9 anos; e 10/11 anos. As variáveis dependentes compreenderam, para além das medidas decorrentes da aplicação da ESI (e.g., deterioração da memória, sugestionabilidade total, submissão e mudança), a avaliação do grau de desejabilidade social das crianças e da sua capacidade de memória fonológica para material verbal não semântico.

Todos os dados recolhidos e respectivos resultados obtidos foram tratados através do programa SPSS (Sta- tistical Package for Social Sciences, versão 19), com um nível de confiança de $95 \%$ e recorrendo à correção de Bonferroni sempre que os pressupostos de aplicação de testes estatísticos assim exigiam. A apresentação dos resultados está seccionada em função dos objetivos e das variáveis consideradas.

\section{Evocação}

Com o objetivo de perceber se o intervalo de retenção (ou momento de evocação) e a idade dos participantes têm efeitos na quantidade de unidades de informação evocadas, aplicamos uma ANOVA 2 X 3 (momento da evocação: imediata $v s$. retardada; idade: $5 / 6$ anos $v s$. $8 / 9$ anos $v s$. 10/11 anos) com medidas repetidas no primeiro fator. Constatamos a existência do efeito principal momento de evocação, $F(1,67)=13,74, p<0,001, \eta^{2}=0,17$. Este dado revela que, independente da idade, o número médio de unidades de informação recordadas é maior no primeiro momento de evocação $\left(M_{\text {evl }}=6,70\right)$ do que no segundo momento $\left(M_{\text {ev2 }}=5,70\right)$. Verificou-se também um efeito principal da idade, $F(2,67)=16,01, p<0,001, \eta^{2}=0,32$. Testes post-hoc de Gabriel revelaram diferenças significativas entre todos os grupos etários $(p<0,01)$. Assim, as crianças com $5 / 6$ anos foram as que apresentaram a média mais baixa de evocação de unidades de informação da história $\left(M_{5 / 6}=2,53\right)$, as crianças de $8 / 9$ anos apresentaram um desempenho intermédio $\left(M_{819}=6,63\right)$ e as crianças de 10/11 anos foram as que recordaram melhor a história $\left(M_{10 / 11}=10,34\right)$, nos dois momentos de evocação. Por fim, não se verificou interação entre o momento de evocação e a idade das crianças, $F(2,67)=0,42, p=0,66, \eta^{2}=0,01$. Os resultados destas análises e respectivos níveis de significância das diferenças entre grupos foram apresentados na Tabela 1.

Tabela 1

Evocação Média dos Grupos Etários em Função do Momento em que Ocorre a Evocação (Desvios Padrões entre Parênteses)

\begin{tabular}{|c|c|c|c|c|c|c|c|c|c|}
\hline \multirow{2}{*}{ Idade } & \multirow{2}{*}{ Evocação 1} & \multirow{2}{*}{ Evocação 2} & \multirow{2}{*}{$F(1,67)$} & \multicolumn{2}{|c|}{$5 / 6 v s .8 / 9$} & \multicolumn{2}{|c|}{$5 / 6$ vs. $10 / 11$} & \multicolumn{2}{|c|}{$8 / 9$ vs. $10 / 11$} \\
\hline & & & & $1^{\mathrm{a}}$ evoc & $2^{\mathrm{a}}$ evoc & $1^{\mathrm{a}}$ evoc & $2^{\mathrm{a}}$ evoc & $1^{\mathrm{a}}$ evoc & $2^{\mathrm{a}}$ evoc \\
\hline $5 / 6$ anos & $2,53(2,27)$ & $1,88(1,90)$ & & & & & & & \\
\hline $8 / 9$ anos & $6,63(4,37)$ & $5,64(4,00)$ & $13,74 * * *$ & $* *$ & $* *$ & $* * *$ & $* * *$ & $* *$ & $*$ \\
\hline 10/11 anos & $10,34(4,71)$ & $9,03(5,07)$ & & & & & & & \\
\hline
\end{tabular}

$* p<0,05 ; * * p<0,01 ; * * * p<0,001$.

\section{Sugestionabilidade}

Analisamos o padrão de sugestionabilidade das crianças tendo em conta a sua idade. No que diz respeito à sugestionabilidade total os dados apontam para a existência de diferenças significativas entre os grupos, $F(2,67)=$ $6,22, p=0,003, \eta^{2}=0,213$. Testes post-hoc de Gabriel aplicados às médias em estudo revelaram que as crianças de 5/6 anos não diferiram das de $8 / 9$ anos quanto ao grau de sugestionabilidade $(p>0,05)$. Contudo, as crianças de 10/11 anos diferiram dos outros dois grupos (10/11 anos $v s .5 / 6$ anos, $p<0,001 ; 10 / 11$ anos $v s .8 / 9$ anos, $p<$ 0,05 ; cf. Tabela 2). Desta forma, podemos verificar que 
Saraiva, M. \& Albuquerque, P. B. (2015). Influência da Idade, Desejabilidade Social e Memória na Sugestionabilidade Infantil.

as crianças de 5/6 anos e de 8/9 anos são mais sugestionáveis do que crianças de 10/11 anos, ou seja, que a sugestionabilidade diminui com o avançar da idade, sendo o seu decréscimo particularmente acentuado a partir dos 10 anos de idade.

Tabela 2

Médias Obtidas nos Indicadores de Sugestionabilidade em Função das Faixas Etárias Estudadas (Desvio Padrão Apresentado entre Parênteses)

\begin{tabular}{ccccc}
\hline Grupo & Submissão 1 & Submissão 2 & Mudança & Sugestionabilidade Total \\
\hline $5 / 6$ anos & $10,13(2,87)$ & $10,50(3,68)$ & $6,50(3,44)$ & $16,63(5,10)$ \\
$8 / 9$ anos & $10,29(2,76)$ & $10,54(2,77)$ & $5,34(2,62)$ & $15,60(3,95)$ \\
$10 / 11$ anos & $7,47(2,93)$ & $8,52(3,22)$ & $4,42(1,95)$ & $11,89(4,42)$ \\
\hline
\end{tabular}

Outra medida de sugestionabilidade que esta escala nos permite obter é o efeito da introdução do feedback negativo na mudança de respostas dadas pelas crianças ao questionário - submissão 2. Para analisar o seu efeito na resposta ao questionário da ESI realizamos uma ANOVA 2 X 3 (medida de submissão: 1 vs. 2; idade: 5/6 anos vs. 8/9 anos vs. 10/11 anos) com medidas repetidas no primeiro fator. Constatamos que a introdução do feedback negativo teve um efeito marginalmente significativo ao nível da submissão, $F(1,67)=3,79, p=0,06, \eta^{2}=0,054$, o que revela uma tendência para todas as crianças aumentarem o número de respostas aquiescentes perante as questões sugestivas, após o feedback negativo. No que se refere ao efeito principal idade os resultados indicaram diferenças significativas entre os grupos, $F(1,67)=5,19, p=0,008$, $\eta^{2}=0,134$. Testes post-hoc de Gabriel aplicados à análise do efeito principal idade revelaram que, as crianças com $10 / 11$ anos $\left(M_{10 / 11}=8,00\right)$ apresentaram uma média de submissão significativamente inferior às dos outros dois grupos $\left(M_{8 / 9}=10,41, p=0,009 ; M_{5 / 6}=10,31, p=0,048\right)$. Não houve efeito de interação entre a idade e a submissão, $F(2,67)=0,78, p=0,46, \eta^{2}=0,023$.

Analisando o efeito do feedback negativo intragrupalmente, através da comparação dos resultados nas medidas de submissão 1 e 2 por idade, verificamos que este efeito foi significativo para o grupo dos 10/11 anos, $t(18)=-2,09$, $p=0,05, d=0,92$. Para os outros dois grupos este padrão de resultados não se replica, $5 / 6$ anos, $t(15)=0,56$, n.s.; $8 / 9$ anos $-t(34)=0,72$, n.s.

Finalmente procedemos à análise dos resultados obtidos pelas crianças na medida de mudança recorrendo a uma ANOVA unifatorial. Os resultados desta análise revelaram que não há diferenças entre os grupos, $F(2,67)=2,62, p=$ 0,08 , ainda que seja notória uma diminuição do seu valor à medida que a idade das crianças avança.

\section{Desejabilidade Social}

Para analisar a associação entre a sugestionabilidade infantil e a desejabilidade social, calculamos a correlação entre os valores obtidos pelas crianças na resposta ao CSD e na medida de sugestionabilidade total da ESI obtendo-se uma correlação de $\mathrm{r}=0,33(p=0,006)$ entre as medidas. Desta forma, um valor mais alto de desejabilidade social está associado a um maior grau de sugestionabilidade interrogativa. De modo a clarificar estes resultados dividimos as crianças em dois grupos tendo em conta a mediana dos seus resultados na escala de desejabilidade social (alta vs. baixa desejabilidade). Aplicamos em seguida um teste $t$ para amostras independentes que revela a existência de diferenças marginalmente significativas entre os dois grupos quanto à sugestionalidade, $t(68)=1,90, p=0,06, d$ $=3,05$, sendo que as crianças que pontuaram mais alto no CSD tendem a ser mais sugestionáveis $\left(M_{\text {CSDelev }}=11,00\right.$; $M_{\text {CSDbaixa }}=6,18$ ).

As nossas análises revelam ainda que a desejabilidade social varia de modo significativo em função do grupo etário, $F(2,67)=6,995, p=0,002, \eta^{2}=0,032$. Os testes post-hoc de Gabriel revelam que o grupo de $5 / 6$ anos $\left(M_{5 / 6}\right.$ $=9,94)$ não diferiu significativamente do grupo $8 / 9$ anos $\left(M_{8 / 9}=9,20\right)$ ao nível do $\operatorname{CSD}(p=0,73)$. No entanto houve diferenças significativas entre o grupo $5 / 6$ anos e o grupo $10 / 11 \operatorname{anos}\left(M_{10 / 11}=6,84 ; p=0,003\right)$ e entre o grupo 8/9 anos e o grupo $10 / 11$ anos $(p=0,007)$. Podemos então constatar que só por volta dos 10/11 anos, as crianças começam a controlar a desejabilidade social da resposta.

\section{Prova de Evocação de Pseudopalavras (PEPp)}

Relativamente à prova de evocação de pseudopalavras, alguma literatura (Candel et al., 2004; Henry \& Gudjonsson, 1999; Scullin et al., 2002) refere que uma maior amplitude de memória fonológica está associada a um menor grau de sugestionabilidade. O nosso estudo aponta no mesmo sentido, pois verificamos que existe uma correlação negativa significativa entre a sugestionabilidade total e a amplitude de memória fonológica, $r=-0,36, p=0,002$.

De modo a compreender melhor a influência da capacidade fonológica na sugestionabilidade infantil, dividimos a amostra em dois grupos em função da mediana do resultado global na prova de pseudopalavras (alta vs. baixa capacidade fonológica). $\mathrm{O} t$-teste para amostras indepen- 
dentes revelou existirem diferenças significativas quanto à sugestionabilidade infantil entre as crianças com maior e menor amplitude de memória fonológica, $t(68)=-2,94, p=$ $0,004, d=2,70$, sendo que crianças com menor amplitude de memória fonológica foram mais sugestionáveis.

\section{Discussão dos Resultados}

A presente pesquisa pretende levar a uma melhor compreensão da expressão da sugestionabilidade infantil, bem como os mecanismos e variáveis que a influenciam em crianças de diferentes grupos etários. Assim sendo, torna-se fundamental estudar e perceber até que ponto os relatos e testemunhos das crianças são credíveis, isto é, até que ponto as crianças são capazes de recordar situações e acontecimentos de modo detalhado e estável, ou se, pelo contrário, as crianças produzem relatos distorcidos, particularmente quando são submetidas a questões sugestivas sobre acontecimentos que recordam com alguma dificuldade.

Por este motivo, a construção e utilização de instrumentos capazes de avaliar a sugestionabilidade infantil tem um papel cada vez mais importante para a compreensão da mesma. $\mathrm{O}$ instrumento estudado neste artigo apresentou a crianças uma história associada a um contexto mais próximo das suas vivências, promovendo assim a recordação de mais pormenores gerais sobre o acontecimento.

Os resultados do presente estudo mostraram, à semelhança do que mais frequentemente é apontado na literatura, uma diminuição da sugestionabilidade infantil com o avançar da idade (Ceci \& Bruck, 1993; Cunha, 2010) Esta diminuição da sugestionabilidade associada ao aumento da idade parece estar relacionada com a melhoria da capacidade geral de recordação das crianças. Ao fazerem relatos cada vez mais detalhados das suas memórias episódicas as crianças aumentam os níveis de certeza e de confiança para estas memórias, diminuindo assim a sua vulnerabilidade à sugestão por terceiros. Carneiro (2007) defende que com a idade a memória episódica se torna progressivamente mais robusta e detalhada por força da utilização adequada de estratégias de recuperação de informação que, e por exemplo, fazem diminuir a produção de memórias falsas.

Contudo a sugestão não tem uma base estritamente mnésica. A maior ou menor dificuldade em lidar com a pressão social exercida por pares ou adultos é também um fator importante no momento de cedência à sugestão. É com o propósito de lidar com esta possibilidade sempre presente nos relatos testemunhados por alguém, que as escalas de sugestionabilidade introduzem o feedback negativo. Os resultados obtidos no nosso estudo mostraram que todas as crianças da nossa amostra são susceptíveis à pressão social exercida, mas que este fator é particularmente relevante até aos 10 anos de idade. É a partir desta idade que a recordação se torna mais rica, a sugestionabilidade se revela mais difícil e a pressão social produz menos mudança nas respostas das crianças. Devemos por isso ter uma particular atenção à obtenção de relatos de crianças com idade inferior a 10 anos. Sublinhe-se que os nossos resultados corroboram o que tem sido referenciado na literatura, nomeadamente que, quando as crianças são sujeitas à mesma questão diversas vezes (na mesma entrevista, ou não), tendem a alterar as suas respostas (Ceci \& Bruck, 1993; Ceci, Crotteau-Huffman, Smith, \& Loftus, 1994). Já no seu estudo, Hunefeldt, Lucidi, Furia e Rossi-Arnaud (2008), alertam para o facto de existir um pico de mudança de resposta após a introdução de feeddback negativo, situado entre os cinco anos e meio e os seis anos de idade. Portanto, a introdução de questões sugestivas repetidas quando pretendemos avaliar a credibilidade/ veracidade de um testemunho infantil deve ser evitada a todo o custo, pois o seu efeito na memória episódica não é dimensionável.

Quanto à desejabilidade social patente nas respostas das crianças, decidimos relacioná-la com a sugestionabilidade a partir da aplicação do CDS (Crandall et al., 1965, versão traduzida por Costa \& Veloso 2008). Costa e Pinho (2010) defendem que a desejabilidade social é inversamente proporcional à idade cronológica, isto é, crianças mais velhas, dão menos respostas socialmente desejáveis do que as crianças mais novas e, consequentemente, um maior grau de desejabilidade social poderá estar associado a um maior grau de sugestionabilidade infantil. A análise dos nossos resultados permite-nos constatar que as crianças mais novas apresentaram desejabilidade social significativamente superior ao das crianças mais velhas, isto é, a tendência para dar respostas de acordo com as expectativas sociais decresce com o avançar da idade. A inexistência de diferenças significativas entre crianças de 5/6 anos e 8/9 anos quanto à desejabilidade social vai ao encontro do padrão geral dos resultados obtidos com a nossa escala, parecendo que, aos 10 anos estamos perante um momento crítico para as variáveis que estamos a analisar: sugestionabilidade e desejabilidade social.

Finalmente, temos também assistido a um debate intenso sobre a relação entre a sugestionabilidade e a capacidade de memória fonológica das crianças. Neste sentido há autores que defendem que quanto maior a capacidade de memória operacional maior a resistência a questões sugestivas (Candel et al., 2004), enquanto outros defendem não existir associação entre as duas variáveis, sobretudo quando a memória é avaliada através de uma prova ou teste, e não pela recordação do material sujeito às questões sugestivas (Cunha, 2010). Reportando-nos aos resultados do presente estudo, verificamos que as crianças mais velhas apresentam um melhor desempenho na tarefa de memória fonológica que lhes foi proposta (recordação de pseudopalavras), o que, como já referimos, é justificável pela sua maior capacidade de memória operacional. Os nossos resultados apoiam assim a proposta de Candel e colaboradores (2004) e realçam a independência da relação encontrada relativamente à idade. Em outras palavras, mesmo entre crianças da mesma idade, aquelas que possuem uma menor capacidade mnésica são mais sugestionáveis do que as crianças que apresentam melhor desempenho na tarefa de memória fonológica. 
Saraiva, M. \& Albuquerque, P. B. (2015). Influência da Idade, Desejabilidade Social e Memória na Sugestionabilidade Infantil.

Concluindo, o presente estudo permitiu alcançar os objetivos a que inicialmente nos propusemos - apresentação de um instrumento de avaliação da sugestionabilidade infantil; compreender a relação da sugestionabilidade infantil com a idade cronológica; avaliar a influência da capacidade mnésica das crianças na resistência a questões sugestivas; e, investigar a relação entre a desejabilidade social e a sugestionabilidade infantil.

Neste sentido, podemos afirmar que após a análise e discussão dos nossos resultados, e em resposta a uma das questões centrais deste trabalho, até aos 10 anos de idade, as crianças são muito sugestionáveis. Com efeito, a introdução de questões sugestivas, que tantas vezes expõem as crianças a informações falsas ou simplesmente distorcidas, revela ser uma fonte de perturbação do relato das crianças, mesmo quando estes ocorreram há muito pouco tempo. Soma-se a este dado que a repetição de questões poder produzir nas crianças a necessidade de mudança das suas respostas, por estas entenderem que a mesma questão feita duas vezes será um indício de que a sua primeira resposta está errada.

Verificamos que as crianças mais novas recordam uma quantidade muito reduzida de unidades de informação, não relatando o acontecimento/história com o mesmo pormenor ou detalhe das crianças mais velhas.

Finalmente importa realçar que há variáveis individuais, como a capacidade de memória fonológica ou a desejabilidade social que estão associadas à sugestionabilidade. No entanto, a sugestionabilidade não se deve meramente a características individuais ou situacionais, mas sim à sua interação (Endres, 1997). Entendemos assim que os profissionais devem estar sensibilizados para o problema que resulta da realização de entrevistas com questões sugestivas, à influência da idade no relato das histórias, à importância do grau de desejabilidade social das crianças na resposta a questões feitas por adultos, bem como aos processos de esquecimento e deterioração da memória episódica.

Se a avaliação da sugestionabilidade infantil é essencial para a credibilidade do sistema de justiça deve ser assumido que o treino de magistrados, policiais, psicólogos e todos os outros entrevistadores de crianças é muito importante para contornar este fenômeno, diminuindo o erro no relato.

Neste sentido, e tendo em conta a relevância deste tema para a sociedade civil, deixamos a sugestão da adaptação da escala construída neste estudo a crianças com deficits e/ou limitações cognitivas, permitindo a compreensão de eventuais diferenças ou semelhanças com as restantes crianças. Por fim, seria útil compreender a influência da introdução de técnicas auxiliares para a evocação da história (e.g., evocação livre seguida de algumas questões que auxiliem as crianças a recordar mais unidades de informação), de modo a verificar se uma melhor memória episódica para a história altera o grau de sugestionabilidade infantil das crianças.

\section{Referências}

Almerigogna, J., Ost, J., Akehurst, L., \& Fluck, M. (2008). How interviewers' nonverbal behaviors can affect children's perceptions and suggestibility. Journal of Experimental Child Psychology, 100, 17-39. doi:10.1016/j.jecp.2008.01.006

Bruck, M., \& Melnyk, L. (2011). Individual differences in children's suggestibility: A review and synthesis. Applied Cognitive Psychology, 25, 202-252. doi:10.1002/acp.1070

Candel, I., Merckelbach, H., Jelicic, M., Limpens, M., \& Widdershoven, K. (2004). Children's suggestibility for peripheral and central details. Journal of Credibility Assessment and Witness Psychology, 5, 9-18.

Carneiro, M. (2007). Memory development in children: What changes with age? Psicologia: Reflexão e Critica, 21, 51-59. doi:10.1590/S0102-79722008000100007

Cassel, W., Roebers, C., \& Bjorklund, D. (1996). Developmental patterns of eyewitness responses to repeated and increasingly suggestive questions. Journal of Experimental Child Psychology, 61, 116-133. doi:10.1006/jecp.1996.0008

Ceci, S., \& Bruck, M. (1993). Suggestibility of the child witness: A historical review and synthesis. Psychological Bulletin, 113, 403-439. doi:10.1037/0033-2909.113.3.403

Ceci, S., \& Bruck, M. (1995). Jeopardy in the courtroom: A scientific analysis of children's testimony. Washington, DC: American Psychological Association.

Ceci, S., Crotteau-Huffman, M., Smith, E., \& Loftus, E. (1994). Repeatedly thinking about non-events: Source misattributions among preschoolers. Conscious and Cognition, 3, 388-407. doi:10.1006/ccog.1994.1022

Ceci, S., \& Friedman, R. (2000). The suggestibility of children: Scientific research and legal implications. Cornell Law Review, 86, 34-108.

Costa, A., \& Pinho, M. (2010). Sugestionabilidade interrogativa em crianças de 8 e 9 anos de idade. Análise Psicológica, 28, 193-208.

Costa, A., \& Veloso, M. (2008). Children's Social Desirability (CSD) - Versão reduzida e adaptada de Crandall, V., Crandall, V., \& Walter, K. (1965). Manuscrito não publicado, Faculdade de Psicologia e Ciências da Educação, Universidade de Coimbra, Portugal.

Crandall, V., Crandall, V., \& Katkovsky, W. (1965). A children's social desirability questionnaire. Journal of Consulting Psychology, 29, 27-36. doi:10.1037/h0020966

Cunha, A. (2010). A sugestionabilidade interrogativa em crianças: O papel da idade e das competências cognitivas (Dissertação de doutoramento, Escola de Psicologia, Universidade do Minho, Braga, Portugal).

Endres, J. (1997). The suggestibility of the child witness: The role of individual differences and their assessment. Journal of Credibility Assessment and Witness Psychology, 2, 44-67.

Goswick, A., Mullet, H., \& Marsh, E. (2013). Suggestibility from stories: Can production difficulties and source monitoring explain a developmental reversal? Journal of Cognition and Development, 14, 607-616. doi:10.1080/15248372.20 12.710864

Gudjonsson, G. (1987). A parallel form of the Gudjonsson Suggestibility Scale. British Journal of Clinical Psychology, 26, 215-221. doi:10.1111/j.2044 8260.1987.tb01348.x

Gudjonsson, G. (1997). The Gudjonsson Suggestibility Scales Manual. Hove, UK: Psychology Press.

Gudjonsson, G., \& Clark, N. (1986). Suggestibility in police interrogation: A social psychological model. Social Behaviour, 1, 83-104. 
Henry, L., \& Gudjonsson, G. (1999). Eyewitness memory and suggestibility in children with mental retardation. American Journal on Mental Retardation, 104, 491-508. doi:10.1352/0895-8017(1999)104

Henry, L., \& Gudjonsson, G. (2003). Eyewitness memory, suggestibility and repeated recall sessions in children with mild and moderate intellectual disabilities. Law and Human Behavior, 27, 481-505. doi:10.1023/A:1025434022699

Hunefeldt, T., Lucidi, A., Furia, A., \& Rossi-Arnaud, C. (2008). Age differences in the interrogative suggestibility of children's memory: Do shift scores peak around 5-6 years of age? Personality and Individual Differences, 45, 521-526. doi:10.1016/j. paid.2008.06.005

Karpinski, A., \& Scullin, M. (2009). Suggestibility under pressure: Theory of mind, executive function, and suggestibility in preschoolers. Journal of Applied Developmental Psychology, 30, 749-763. doi:10.1016/j.appdev.2009.05.004

Liebman, J., McKinley-Pace, M., Leonard, A., Sheesley, L., Gallant, C., Renkey, M., \& Lehman, E. (2002). Cognitive and psychosocial correlates of adults' eyewitness accuracy and suggestibility. Personality and Individual Differences, 33, 49-66. doi:10.1016/S0191-8869(01)00135-0

Lobo, F., Acrani, I., \& Ávila, C. (2008). Tipo de estímulo e memória de trabalho fonológica. Revista CEFAC, 10, 461-470.

Loftus, E., \& Palmer, J. (1974). Reconstruction of automobile destruction: An example of the interaction between language and memory. Journal of Verbal Learning and Verbal Behavior, 13, 585-589. doi:10.1016/S0022-5371(74)80011-3

Okanda, M., Kanda, T., Ishiguro, H., \& Itakura, S. (2013). Threeand 4-year-old children's response tendencies to various interviewers. Journal of Experimental Child Psychology, 116, 68-77. doi:10.1016/j.jecp.2013.03.012

Pickering, S., \& Gathercole, S. (2001). Working Memory Test Battery for Children (WMTB-C). London: Pearson Assessment.

Principe, G., \& Schindewolf, E. (2012). Natural conversations as a source of false memories in children: Implications for the testimony of young witnesses. Developmental Review, 32 , 205-223. doi:10.1016/j.dr.2012.06.003
Roediger, H., \& McDermott, K. (1995). Creating false memories: Remembering words not presented in lists. Journal of Experimental Psychology: Learning, Memory, and Cognition, 21, 803-814. doi:10.1037/0278-7393.21.4.803

Saraiva, M., \& Albuquerque, P. (2012). Escala de Sugestionabilidade Interrogativa para Crianças (ESI). Manuscrito não publicado, Laboratório de Cognição Humana, Universidade do Minho, Braga, Portugal.

Schacter, D. (1999). The seven sins of memory: Insights from psychology and cognitive neuroscience. American Psychologist, 54, 182-203. doi:10.1037//0003-066X.54.3.182

Schacter, D. (2003). Richard Semon. In J. H. Byrne (Ed.), Encyclopedia of learning and memory. New York: Macmillan.

Scullin, M., \& Ceci, S. (2001). A suggestibility scale for children. Personality and Individual Differences, 30, 843-856. doi:10.1016/S0191-8869(00)00077-5

Scullin, M., Kanaya, T., \& Ceci, S. (2002). Measurement of individual differences in children's suggestibility across situations. Journal of Experimental Psychology: Applied, 8, 233-246. doi:10.1037/1076-898X.8.4.233

Stein, L., Pergher, G., \& Feix, L. F. (2009). Desafios da oitiva de crianças e adolescentes: Técnica de entrevista investigativa. Brasília, DF: Secretaria Especial dos Direitos Humanos da Presidência da República.

Vicente, S. (2003). Reconhecimento de palavras faladas: Abordagem desenvolvimental em português europeu (Vols. 1-2) (Dissertação de doutoramento, Faculdade de Psicologia e Ciências da Educação, Universidade do Porto, Portugal).

Welter, C., \& Feix, L. (2010). Falsas memórias, sugestionabilidade e testemunho infantil. In L. M. Stein (Ed.), Falsas memórias. Fundamentos científicos e suas aplicações clínicas e jurídicas (pp. 157-185). Porto Alegre, RS: Artmed.

\section{História da Escala de Sugestionabilidade Interrogativa (Saraiva \& Albuquerque, 2012)}

As unidades de informação estão separadas por barras.

O André e o Simão Almeida / são irmãos / e moram em Lisboa. / São dois rapazes atléticos, / que adoram andebol, / e jogam no Sporting. / Vivem com os pais, / numa pequena casa,/ que tem um jardim, / onde o seu cão Balú/ tem a sua casota./ O André anda na escola primária / e o Simão na secundária / onde a mãe deles trabalha./

Uma quarta-feira / de manhã, / em Março, / os irmãos saíram de casa, / para a escola,/ e no percurso ouviram /junto ao passeio / um telemóvel a tocar. / Os irmãos / ficaram sem saber o que fazer, / mas decidiram atender o telemóvel. / Ouviram então alguém / a dizer que se chamava Rita, / e que queria falar com a Joana. / Os rapazes / explicaram que tinham encontrado o telemóvel,/ no meio da rua/ e que gostariam de o entregar à dona/. A Rita/ explicou que a Joana/ andava na escola secundária/ Vasco Botelho. /Esta era também a escola do Simão./ Ao chegar à escola/ o Simão procurou a Joana/, da turma $10^{\circ} \mathrm{J} / \mathrm{e}$ entregou-lhe o telemóvel/. Passados quatro anos/ sobre este episódio/ a Joana e o Simão ainda são amigos. 